major threats to carnivores and the challenges to their conservation in this region and to provide recommendations for the protection of biodiversity in the central High Atlas. The workshop was an opportunity to reflect on past conservation studies and actions and to discuss coordination among researchers, local people, regional and national authorities, and NGOs.

The carnivore species of the High Atlas are threatened by overhunting, habitat destruction, highly fragmented populations and the risk of local extinction. Surveys of carnivores in the central High Atlas during 2019-2021 by AE and AF, with support from The Rufford Foundation, indicated there is increasing conflict between people and wild carnivores, particularly in the context of the predation of domestic animals. The majority of inhabitants have negative attitudes towards wild carnivores, and hunt and kill them to protect their livestock. Although the inhabitants know it is illegal to capture or kill wild carnivores, they continue to do so, using techniques such as poisoning.

To conserve the native carnivores of the central High Atlas, the workshop participants agreed that urgent measures need to be taken to (1) encourage rural community development projects, (2) help the inhabitants use non-lethal techniques to protect their livestock from carnivores, (3) manage human-carnivore interactions to increase public tolerance for wild carnivores, (4) raise awareness about the ecological and economic roles of wild carnivores, and (5) develop compensation programmes to assist local people by reimbursing them for losses attributable to predators.

ABDERRAZAK El Alami (10 orcid.org/0000-0002-0274-1430, departementbiologiefssm@hotmail.com) Khawarezmi High School, Academy of Education and Training of Beni Mellal-Khenifra, Ministry of National Education, Vocational Training, Higher Education and Scientific Research, Morocco. ADERRAZZAK FATTAH (10 orcid.org/0000-0003-1146-0291) Laboratory of Bio-Geosciences and Materials Engineering, Higher Normal School, University of Hassan II, Casablanca, Morocco

This is an Open Access article, distributed under the terms of the Creative Commons Attribution licence CC BY 4.0.

\section{Beliefs that Indian pangolins have mystical powers threaten the species}

The Eastern Ghats are a discontinuous chain of mountain ranges in South India. The Indian pangolin Manis crassicaudata, categorized as Endangered on the IUCN Red List, inhabits these human-dominated landscapes, especially where there is an abundant supply of ants and termites. A survey during August 2020-July 2021 of households in 21 villages in the region, outside protected areas, revealed that promulgation, through social media, of local beliefs that pangolins can produce electricity, is leading to increased demand for pangolin scales, and may be contributing to a decline in this species across the region.

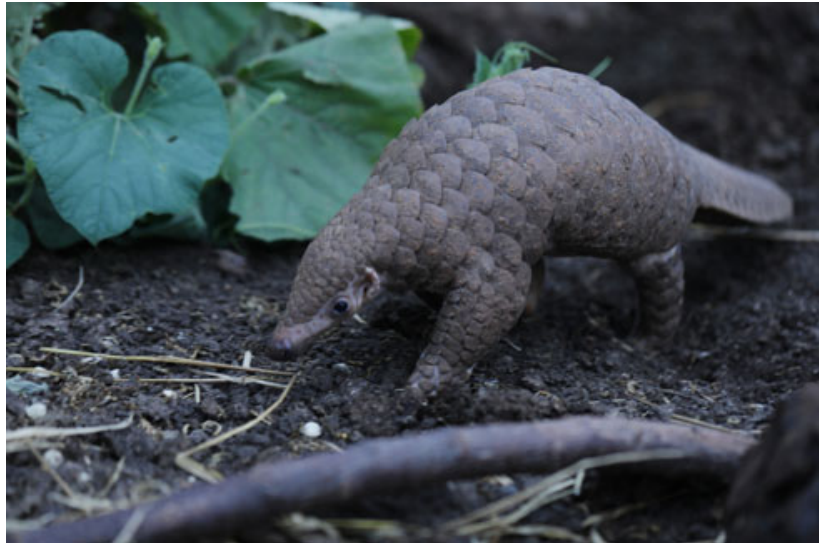

An Indian pangolin rescued from a village by the Eastern Ghats Wildlife Society. Photo: Appanna Saragada.

Our survey revealed that the high economic value of the scales has resulted in increased hunting and trapping of pangolins. The scales are sold for USD 500-1,000 per $\mathrm{kg}$ on the local black market, and are used to make pendants to deter evil spirits, although this use is not apparently prevalent amongst the younger generation. It was also reported to us that some remote tribal communities use the scales as earrings, and that pangolin meat is consumed in some areas.

There are claims that pangolins produce a mysterious energy and can emit electrical sparks when the keratinised scales are probed with a testing screwdriver. Videos of the light of a testing screwdriver glowing when used to probe a pangolin's scales have been circulating on various social media platforms. Pangolins do not generate electricity and the screwdriver was presumably rigged to produce the glow. Our interviews also revealed another popular myth about pangolins: that forest fires can be caused by a pangolin rubbing its scales against dry grass or leaf litter in summer.

Beliefs and myths about the pangolin's mystical powers and medicinal value are part of the culture of the Eastern Ghats, and will need to be addressed as part of conservation efforts to halt the illegal trade in this species. Although education and awareness programmes can help instruct people about the ecological significance and conservation status of the Indian pangolin, tailored behaviour change campaigns through community outreach and engagement are required, along with proactive law enforcement measures, to influence behaviour and decisionmaking processes for reduction of anthropogenic pressures on the Indian pangolin in the Eastern Ghats.

We thank the Andhra Pradesh Forest Department and Save Pangolins for their support and encouragement.

\section{MuRTHY Kantimahanti (@ orcid.org/0000-0002-4761-8583, klnmurthy25@gmail.com) Eastern Ghats Wildlife Society, Vepagunta, Visakhapatnam, India}

This is an Open Access article, distributed under the terms of the Creative Commons Attribution licence CC BY NC SA 4.0. 